Efecto de la Asignación Universal por Hijo en la reducción de disparidades regionales en Argentina

Darío Rossignolo 
Lecturas de Economía, 96 (enero-junio 2022), pp. 71-99

Darío Rossignolo

\section{Efecto de la Asignación Universal por Hijo en la reducción de disparidades regionales en Argentina}

Resumen: En el marco de un incremento significativo en los niveles de gasto público, a partir de 2009 Argentina instrumentó la Asignación Universal por Hijo, un programa de transferencia de ingresos de diseño universal sujeto al cumplimiento de determinadas condicionalidades. Este programa ha tenido efectos en la reducción de disparidades de ingresos, pero el efecto regional no ha sido analizado por la literatura. Utilizando la metodología de diferencias dobles, en este articulo se presentan estimaciones que procuran mostrar si el programa ha tenido efectos también en la reducción de las brechas regionales en términos de desigualdades de ingresos, pobreza e indigencia. Los resultados muestran que, si bien pueden apreciarse algunos efectos de reducción de disparidades, no puede asegurarse que las brechas regionales reflejadas en la Encuesta Permanente de Hogares (EPH) disminuyan en forma estadísticamente significativa en todos los casos.

Palabras clave: desigualdad, transferencias monetarias, brechas regionales, pobreza, dobles diferencias.

Clasificación JEL: H53, H73, H22.

\section{The Effect of "Asignación Universal Por Hijo" in the Reduction of Regional Disparities in Argentina}

Abstract: Consistently with the substantial increase in public expenditure levels, in 2009 Argentina instrumented a public cash transfer program, called Asignación Universal por Hijo, which had been designed as a universal program, but subject to the fulfillment of several requirements. This program has had an effect on the reduction in income disparities, but the regional effect has not been analyzed by the literature. By employing the methodology known as differences in differences, this paper presents estimations that attempt to show whether this transfer has been effective in reducing regional differences in terms of income inequality, and both moderate and extreme poverty, Results show that although there are several effects on the reduction of disparities, it cannot be assured that all regional gaps reflected in $E P H$ are reduced in a statistically significant way.

Keywords: inequality, monetary transfers, regional disparities, poverty, double differences.

https://doi.org/10.17533/udea.le.n96a345762

\section{(cc) BY-NC-SA}

Este artículo y sus anexos se distribuyen por la revista Lecturas de Economía bajo los términos de la Licencia Creative Commons Atribución-NoComercial-CompartirIgual 4.0. https://creativecommons.org/licenses/by-nc-sa/4.0/ 


\section{Effet de l'Allocation Universelle pour Enfants sur la réduction des disparités régionales en Argentine}

Résumé: Dans le cadre d'une angmentation significative des niveaux de dépenses publiques, depuis 2009, l'A 'rgentine a mis en cuvre l'Allocation universelle pour enfants, un programme de transfert de revenus universellement conçu sous réserve du respect de certaines conditions. Ce programme a eu des effets sur la réduction des disparités de revenu, mais l'effet régional n'a pas été analysépar la littérature. En utilisant la méthodologie de la double différence, cet article présente des estimations qui visent à montrer si le programme a également eu un impact sur la réduction des écarts régionaux en termes d'inégalités de revenus, de pauvreté et d'indigence. Les résultats montrent que, bien que certains effets de réduction des disparités puissent être observés, il n'est pas certain que les écarts régionaux reflétés dans l'Enquête permanente auprès des ménages (EPH) diminueront statistiquement de manière significative dans tous les cas.

Mots clés: inégalité, transferts monétaires, écarts régionaux, pauvreté, doubles différences

Cómo citar / How to cite this item:

Rossignolo, D. (2022). Efecto de la Asignación Universal por Hijo en la reducción de disparidades regionales en Argentina. Lecturas de Economia, 96, 71-99.

https://doi.org/10.17533/udea.le.n96a345762 


\title{
Efecto de la Asignación Universal por Hijo en la reducción de disparidades regionales en Argentina
}

\author{
Darío Rossignolo $\circledast^{\mathrm{a}}$
}

-Introducción. -I. Evolución normativa y cuantitativa. -II. Revisión de literatura. -III. Descripción de la metodología. -IV. Impacto de la AUH en los indicadores seleccionados -Conclusiones. -Agradecimientos. -Referencias.

Primera versión recibida el 30 de marzo de 2021; versión final aceptada el 10 de agosto de 2021

\section{Introducción}

En el año 2009, Argentina instrumentó un programa de transferencias de ingresos que tiene el objetivo de mejorar la calidad de vida y el acceso a la educación de niños, niñas y adolescentes denominado Asignación Universal por Hijo. La población objetivo está constituida por las familias con hijos menores de 18 años que se encuentren desocupados o se desempeñen en la economía informal, con un ingreso inferior al salario mínimo del sector formal. Es un programa de carácter nacional; el método de focalización se realiza mediante una comprobación de medios indirecta.

Este tipo de programas de transferencia pública tiene una gran variedad de efectos, muchos de los cuales han sido analizados por la literatura. Numerosos han sido los estudios que han analizado el impacto de la Asignación Universal por Hijo sobre la desigualdad en la distribución del ingreso personal y la pobreza (Maurizio, 2009 Lustig \& Pessino, 2013, Rossignolo, 2016), y en relación con la fecundidad, oferta de trabajo y requerimientos educativos (Garganta \&Gasparini, 2012; Maurizio \& Vázquez, 2014; Garganta et al., 2015; Garganta et al., 2017; Edo et al., 2015; Edo \& Marchionni, 2018). Los resultados de estos estudios señalan la importancia de la Asignación

a Darío Rossignolo: profesor de la Universidad de Buenos Aires, Facultad de Ciencias Económicas, Buenos Aires, Argentina. Profesor de la Universidad Nacional de Córdoba, Facultad de Ciencias Económicas, Córdoba, Argentina. Dirección electrónica: darossignolo@economicas.uba.ar https://orcid.org/0000-0001-7960-8841 
Rossignolo: Efecto de la Asignación Universal por Hijo en la reducción de disparidades...

Universal por Hijo en la reducción de la pobreza y la desigualdad de ingresos, así como - también — el efecto positivo en la fecundidad y escolarización, sin registrarse desincentivos estadísticamente significativos sobre la oferta de trabajo de los adultos en general y de las mujeres en particular.

Sin embargo, la dimensión regional de esta transferencia ha sido poco explorada. Los trabajos de Agiset al. (2013) y Roca (2011), constituyen los antecedentes más relevantes. En los mismos se enfatiza el efecto en la disminución de la pobreza y la desigualdad de ingresos del programa para cada una de las regiones de Argentina.

Este enfoque, si bien no ha sido investigado en gran medida por la literatura, cobra particular relevancia al analizarlo desde el punto de vista del federalismo fiscal, dado que uno de los objetivos de las transferencias llevadas a cabo por el nivel nacional a las regiones se relaciona con el principio de igualdad de oportunidades o igualdad de provisión de ciertos bienes públicos, además de la igualación de los residuos fiscales. Los gobiernos locales tienen jurisdicción no solo sobre grupos de personas con preferencias disímiles sino también sobre territorios con distintas bases imponibles y distintos costos de provisión de los servicios públicos. Existen regiones con ingreso per cápita promedio alto, y regiones con ingreso bajo, que están compuestas tanto por individuos de ingreso alto como por individuos de ingreso bajo, que habitan indistintamente en ambas regiones, aunque en diferente proporción. La Asignación Universal por Hijo es una transferencia federal focalizada en los individuos más pobres; si éstos conforman en una mayor proporción a las regiones más pobres, la AUH podría estar ayudando a reducir las brechas regionales de pobreza y desigualdad en la distribución del ingreso, entre otros indicadores relevantes.

Por ejemplo, el ingreso medio per cápita real de las regiones del Noroeste (NOA) y del Noreste (NEA) representan-a partir de la información de la Encuesta Permanente de Hogares (EPH)del Instituto Nacional de Estadística y Censos de Argentina (INDEC)—, en promedio para los períodos 2004 a 2016, entre el $47 \%$ y el $53 \%$ del ingreso medio per cápita real de la región Patagónica. Las regiones del NOA y NEA — entretanto- resultan estar entre las de menor desigualdad del país, inferior inclusive a la desigualdad existente 
en el Gran Buenos Aires (GBA). En términos de pobreza e indigencia la región Patagónica muestra niveles casi tres veces inferiores a la región del NEA, mientras que la región del GBA evidencia magnitudes menores a las del NEA en —aproximadamente- el 50\%. Dado que la AUH consiste en una transferencia nacional cuyos beneficiarios se encuentran en las diferentes regiones geográficas, es interesante establecer si la misma ha resultado de utilidad a los fines de reducir las disparidades regionales en las variables mencionadas reflejadas a partir de la información de la Encuesta Permanente de Hogares en las variables mencionadas.

Para este fin se utilizará como estrategia empírica un modelo de diferencias en diferencias a partir de los datos de la Encuesta Permanente de Hogares. Este mide el efecto de un tratamiento en un determinado período en el tiempo y representa la diferencia entre el pre y post tratamiento, dentro de los grupos de tratamiento y control, para las diferentes regiones de Argentina.

En el modelo se comparan los resultados en términos de ingreso per cápita, desigualdad, tasas de pobreza e indigencia, antes de la instrumentación de la AUH y después de la recepción de esta por parte de los hogares, en varias ondas temporales sucesivas, lo que permitiría evaluar la evolución de las brechas regionales relacionadas con los referidos indicadores. En consecuencia, el aporte de este artículo consiste en la aplicación de la metodología de diferencias en diferencias para analizar si la instrumentación de la AUH resultó de utilidad para reducir las brechas regionales en los indicadores mencionados.

La estructura del artículo es la siguiente: en el primer apartado se presentará una descripción normativa del programa de transferencia monetaria, junto con su evolución cuantitativa en términos presupuestarios y de alcance de cobertura; en segundo lugar, se expondrá un breve relevamiento de la literatura sobre el análisis del impacto de este tipo de programas. En tercera instancia se presentará el modelo a desarrollar, mientras que en la cuarta sección se explicitarán los resultados de las estimaciones para el análisis de las brechas regionales. La última sección introduce las conclusiones. 
Rossignolo: Efecto de la Asignación Universal por Hijo en la reducción de disparidades...

\section{Evolución normativa y cuantitativa}

\section{A. Una mirada global}

A partir del año 2008, la evolución del gasto público social en Argentinaha tenido como uno de sus principales objetivos sostener el nivel de ingresos y empleo amenazado por la crisis económica internacional. Dentro de ese marco se incluye la creación de la Asignación Universal por Hijo (AUH), que implicó la extensión de los beneficios de la asignación por hijo que el Estado otorga a los trabajadores que se desempeñan en el sector formal de la economía, a los niños con padres que sean trabajadores informales o del servicio doméstico cuyo ingreso sea inferior al salario mínimo, vital y móvil, o desocupados que no perciban seguro por desempleo.

En cuanto a la evolución cuantitativa, el gasto público total en Argentina en todos los niveles jurisdiccionales pasó del $26 \%$ del PIB en 2004 a alrededor del $47 \%$ PIB en 2015. Considerando solo el gasto público social —y restringiendo la comparación al período comprendido entre 2009 y $2016-$ se aprecia que Argentina experimentó un marcado crecimiento en el mismo, no solo en el gasto social total sino también en lo que corresponde a las transferencias monetarias. Para 2010 el gasto social en Argentina representaba el $26 \%$ del PIB, aumentando al 30,8\% en 2016. Estos niveles de gasto, que significaban en ambos períodos los valores más elevados de América Latina - superiores inclusive a los valores de Brasil, de 23,4\% y 25,5\%, respectivamente para cada uno de los períodos_-, también resultaban elevados en 2010 en comparación con la mayoría de los países de la OCDE, y lo colocaba solo ligeramente por debajo de Francia en 2016.

Cuando se analizan las transferencias monetarias como porcentaje del PIB (Producto Interno Bruto), en Argentina representaban en 2010 el 12\% del PIB, por debajo del promedio de la OCDE para 2010. En 2015, sin embargo, las mismas representaban el $15 \%$ del PIB, bien por encima del promedio y muy cercano a los valores de gasto más elevados en relación con los países de la OCDE. 


\section{B. La Asignación Universal por Hijo}

Es un programa de transferencias de ingresos con carácter universal que tiene el objetivo de mejorar la calidad de vida y el acceso a la educación de niños, niñas y adolescentes. Se basa en un enfoque de protección social, dado que intenta equiparar a los trabajadores informales con los asalariados formales, cuyos hijos están cubiertos por la Asignación Familiar Contributiva. La población objetivo son las familias con hijos menores de 18 años y mujeres embarazadas que se encuentren desocupadas o se desempeñen en la economía informal con un ingreso inferior al salario mínimo del sector formal, empleados domésticos con ingresos menores al salario mínimo.

El máximo de prestaciones a percibir es acumulable al importe equivalente a cinco menores. El $80 \%$ del monto previsto se abona mensualmente a los titulares del beneficio y el $20 \%$ podrá cobrarse cuando el titular acredite el cumplimiento de las condicionalidades de salud y educación.

La condicionalidad para los menores de 5 a 18 años consiste, en el caso de educación, en cumplir con la asistencia regular a un establecimiento educativo formal reconocido por el Ministerio de Educación. En cuanto a salud, deben tener el esquema de vacunación completo o en curso para la edad. La percepción de esta prestación resulta incompatible con el cobro de cualquier suma originada en prestaciones contributivas o no contributivas nacionales, provinciales o municipales, y dado que su beneficio resulta en un monto mayor al correspondiente a los otros planes de transferencias condicionadas y a la inscripción del perceptor como contribuyente del Régimen Simplificado para Pequeños Contribuyentes, aunque este requisito fue eliminado a partir de 2017.

En consecuencia, la principal razón por la cual se utilizó el período 2004-2016 para el análisis de este artículo estriba en el hecho de que a partir de 2017 se eliminan las incompatibilidades con la percepción de otros programas nacionales o provinciales para poder percibir la AUH, junto con el hecho de permitir que los perceptores que estuvieran inscriptos en el Régimen Simplificado para Pequeños Contribuyentes puedan ser elegibles, lo que estaba vedado antes de esa fecha. Esto corresponde a un cambio en la normativa que dificultaría la comparación en los períodos posteriores a la periodización elegida. 
Rossignolo: Efecto de la Asignación Universal por Hijo en la reducción de disparidades...

Dado que la percepción de esta prestación resultaba incompatible con el cobro de cualquier suma originada en prestaciones contributivas o no contributivas nacionales, provinciales o municipales — hasta 2016- y dado que su beneficio resulta en un monto mayor al correspondiente a los otros planes de transferencias condicionadas, el programa presupone la desaparición del Programa Jefes y Jefas de Hogar Desocupados (PJ y JHD) y el Programa Familias para la Inclusión Social(PFIS), ambos surgidos luego de la crisis de 2002. La creación de la AUH en 2009 generó el traspaso de muchos de los beneficiarios a la percepción de dicha transferencia.

En términos de cobertura, debe especificarse que la cantidad de beneficiarios del programa ha sido creciente. En 2009 los beneficiarios alcanzados fueron 3,4 millones de personas (1,8 millones de hogares), mientras que hacia 2016 el programa abarcaba 3,9 millones de beneficiarios (1,9 millones de hogares). Presupuestariamente, la AUH rondó en el período analizado el 0,5\% del PIB, lo que representaba — por ejemplo, en órdenes de magnitud - el 50\% del déficit primario para 2015, o la tercera parte del déficit de 2016.

\section{Revisión de literatura}

Los estudios que han analizado el impacto de la Asignación Universal por Hijo se han focalizado - principalmente- en el análisis del efecto de esta sobre la desigualdad en la distribución del ingreso, la pobreza, la fecundidad y la oferta de trabajo, entre otros factores. La literatura sobre la temática se divide en tres grupos, a saber: los trabajos que analizan el impacto sobre pobreza y desigualdad mediante una metodología de "incidencia presupuestaria"; aquellos que estudian el impacto sobre fecundidad y oferta de trabajo empleando un análisis de diferencias dobles, y un tercer grupo que investiga el impacto regional utilizando una metodología similar al primero de los grupos analizados.

El primer trabajo sobre esta temática, que planteaba la instrumentación de esta política cuando la misma aún no había sido llevada a cabo, es el de Maurizio (2009. En el mismo, enrolado en el primero de los grupos, se evalúan diferentes esquemas de transferencias monetarias a los hogares con el 
objetivo de reducir los niveles de pobreza, indigencia y desigualdad simulando tanto transferencias a los hogares con presencia de menores excluidos del sistema contributivo de asignaciones familiares, como a los desocupados sin seguro de desempleo, o a los adultos mayores sin percepción jubilatoria, evaluando el impacto y el costo de las diferentes alternativas. En todos los casos mencionados las estimaciones de los efectos de las políticas presentadas reflejan la consecución del doble objetivo de reducir la desigualdad y la pobreza.

Utilizando también un análisis de incidencia fiscal, Lustig y Pessino (2013) y Rossignolo (2016) muestran el impacto de la AUH sobre la desigualdad y pobreza al estudiar el impacto del conjunto de los impuestos y los gastos públicos, determinando que la AUH y el resto de los programas de transferencia monetaria tienen un marcado efecto de progresividad en la distribución del ingreso y de reducción de los niveles de indigencia y pobreza.

A similares conclusiones, también mediante la metodología de incidencia presupuestaria, llegan Higgins y Pereira (2013), quienes analizan el caso del programa Bolsa Familia para el caso de Brasil, y Scott et al. (2017) con el Programa de Educación, Salud y Alimentación para México. En ambos casos los programas ayudan a reducir la proporción de personas por debajo de las líneas de pobreza y la desigualdad en la distribución del ingreso.

Entre los trabajos incluidos en el segundo de los grupos, merecen citarse a Garganta y Gasparini (2012), quienes estudian el efecto del programa sobre la formalidad laboral, comparando en el tiempo grupos de individuos beneficiados y los no elegibles — trabajadores informales y desocupados con y sin hijos- L Los resultados muestran un desincentivo a la formalidad estadísticamente significativo.

Maurizio y Vázquez (2014) utilizan una metodología de estimador de diferencias en diferencias en conjunto con técnicas de emparejamiento por índices de propensión para evaluar los efectos a corto plazo del programa en la participación laboral de los adultos y en la generación de ingresos. Los resultados sugieren que, en el primer año de operación analizado, el programa no desalentó el trabajo de los adultos ni originó una reducción del número de horas trabajadas. 
Rossignolo: Efecto de la Asignación Universal por Hijo en la reducción de disparidades...

Garganta et al. (2015), también mediante la metodología de diferencias en diferencias, estudian el impacto de la AUH sobre la participación laboral femenina comparando en el tiempo los resultados laborales entre mujeres elegibles y no elegibles. Los resultados sugieren un efecto negativo y significativo del programa sobre la participación laboral de las mujeres. No se encuentra evidencia de un efecto sobre la oferta laboral de los hombres.

Garganta et al. (2017) investigan el potencial efecto de la AUH en las decisiones de fertilidad a través de un modelo de diferencias en diferencias. Las variables de control incluyen edad, edad al cuadrado, estado civil, nivel educativo, número de hijos, región geográfica y dummies temporales. Los resultados sugieren un impacto positivo y significativo en los hogares con al menos un hijo, pero sin efectos significativos en hogares sin hijos.

Edo et al. (2015) estudian la evolución del cumplimiento de los requisitos educativos obligatorios a partir de la instrumentación de la AUH. Utilizando un modelo de diferencias dobles estiman que la participación en el programa impacta positivamente en el incremento de la escolaridad de los niños elegibles entre 15 y 17 años e incluyen un set de controles a nivel individual y a nivel de hogar. Entre los primeros pueden citarse, por ejemplo, la edad y edad al cuadrado de los niños y del jefe de hogar, su sexo, nivel educativo y condición de ocupación. Mientras tanto, entre los controles a nivel de hogar se encuentran la cantidad de miembros, ingreso per cápita familiar, hogares monoparentales, hogares con jefatura femenina y cantidad de menores de 18 años.

Como fuera expresado con anterioridad, el impacto regional de la AUH ha sido poco explorado. Cont y Porto (2016) estudian el impacto del presupuesto público, en forma global, sobre la distribución personal y regional del ingreso. Agis et al. (2013) y Roca (2011) desarrollan un análisis para determinar el impacto de la AUH sobre los indicadores de pobreza, indigencia, desigualdad y vulnerabilidad relativa —en el caso de Agis et al. (2013) - utilizando los microdatos de la Encuesta Permanente de Hogares (EPH), los índices de precios para las canastas básicas de consumo del GBA y del resto del país y datos provenientes de registros administrativos, verificando, entre otros resultados, que todos los indicadores analizados muestran una mejora 
significativa, especialmente en las regiones más carenciadas del país, como el norte argentino.

Este artículo difiere de los estudios anteriores en que analiza el impacto de la AUH en la disminución de las brechas regionales para un conjunto de indicadores significativos como pobreza, indigencia, desigualdad e ingreso por habitante.

\section{Descripción de la metodología}

Para identificar el potencial efecto causal de la Asignación Universal por Hijo sobre el ingreso se aplica la metodología de diferencias en diferencias o diferencias dobles, utilizando los microdatos de la EPH, el principal relevamiento del país en temas laborales y de ingresos queabarca los principales aglomerados urbanos —más de 100000 habitantes_ del país. Estos aglomerados están comprendidos en seis regiones a saber: Gran Buenos Aires (GBA), Región Noroeste (NOA), Región Noreste (NEA), Cuyo, Región Patagónica y Región Pampeana. Releva información sobre ingresos, ocupación y condición de formalidad, nivel educativo, entre otros. Todas las estimaciones de este trabajo y los resultados obtenidos se circunscriben a la información de esta encuesta y no deben extrapolarse al universo poblacional; las disparidades regionales que se procuran evaluar en este artículo son las que se obtienen a partir del procesamiento de la información de la propia encuesta ${ }^{1}$.

La elección de esta metodología no experimental se debe - fundamentalmente- a que la asignación al tratamiento del programa no fue realizada de manera aleatoria sobre la población —-metodología de intención al tratamiento, ver Garganta et al.(2015)_- En este sentido, la estrategia de identificación consiste en compararen el tiempo, es decir, antes y después de la política, las diferencias existentes en un conjunto de indicadores entre el grupo de tratamiento y el grupo de control para los diferentes pares de regiones. Estos indicadores son: el índice de Gini de desigualdad en la distribución del

1 Esto implica que, si existieren regiones con grupos poblacionales no alcanzados por la encuesta, los efectos de la política no se verían reflejados en los resultados de las estimaciones de este artículo. 
Rossignolo: Efecto de la Asignación Universal por Hijo en la reducción de disparidades...

ingreso, la proporción de individuos pobres e indigentes, y el nivel de ingreso per cápita de cada una de las regiones identificadas en la EPH.

Como la EPH no permite distinguir a los beneficiarios del programa, la metodología consiste en imputar los montos correspondientes a la transferencia monetaria bajo análisis a los hogares a los que le corresponda percibirla de acuerdo con sus características. Para cada onda trimestral de la EPH comprendida entre el primer trimestre de 2004 y el cuarto trimestre de 2016 se simularon las condiciones teóricas necesarias para que cada hogar fuera perceptor de la mencionada transferencia monetaria ${ }^{2}$. Esta metodología, tradicional de los estudios de incidencia, es la de benefitincidence analysis. En Higgins y Lustig (2017) se explica detalladamente este procedimiento denominado simulation method ${ }^{3}$. Tal como se realiza en los ejercicios tradicionales de incidencia, se asume que la totalidad de los beneficiarios se distribuye de la misma manera que los beneficiarios relevados en las encuestas ${ }^{4}$.

Se define en consecuencia un grupo de "tratamiento" y otro de "control" basado en su elegibilidad, la que está definida a su vez por la condición laboral de los padres en el mercado de trabajo (Edo et al., 2015; Garganta, et al., 2015). Por este motivo, el grupo de tratamiento incluye a los hogares elegibles por la AUH de acuerdo con los criterios y condiciones estipulados paraser beneficiario —informales con hijos menores a 18 años, entre otros-, una vez que se estima que han recibido el programa.

Existe en el cuestionario de la EPH una pregunta que se utiliza para identificar informalidad. En ella se les consulta a los trabajadores asalariados si han sido objeto de deducciones al salario percibido en sus trabajos con

$\overline{2}$ Se asume que estas transferencias han sido financiadas mediante recaudación de impuestos no incluidos en la información de la encuesta, como impuestos sobre consumos o patrimonios.

3 Allí se menciona que, para el caso de Argentina, mediante chequeos de robustez no se encontraron diferencias significativas al comparar con el caso de existencia de filtraciones en la focalización de las transferencias.

4 Como extensión al trabajo podría evaluarse el caso en el que la distribución de los beneficiarios efectivos no coincida con los teóricos; para ello sería relevante contar con la información de los perceptores efectivos del programa de transferencia monetaria y su distribución a lo largo de los tramos de ingreso. 
el fin de realizar aportes al sistema de seguridad social. Esto implica que cualquier trabajador que se encuentre en esta condición no calificaría para la AUH —sí podría hacerlo, en su caso, para las Asignaciones Familiares Contributivas-. El grupo de control, en consecuencia, está compuesto por los hogares con empleo formal —es decir, los hogares que responden positivamente a la pregunta anterior-, los que se asume que conservan similares características antes y después de la instrumentación de la AUH. La contrastación entre estos dos grupos - el grupo de tratamiento y el grupo de control— en el tiempo — antes y después de la AUH— permitirá estimar el efecto potencial ex-post de la política sobre los ingresos de los hogares.

La metodología empleada utiliza un modelo OLS, en donde la ecuación inicial es:

$$
Y_{i t}=\alpha+\beta D_{i}+\gamma X_{i t}+\varepsilon_{i t}
$$

Las variables dependientes $Y_{i t}$ determinan, en cada caso, los valores de desigualdad, pobreza, indigencia, ingreso per cápita y cantidad de niños asistentes a la escuela pública para la región $i$ en cada período $t$. En este caso $D_{i}$ es una variable dummy que toma el valor 1 si la observación está en el grupo de "tratamiento" y el valor 0 si está en el grupo de "control”. La matriz $X_{i t}$ representa las variables de control y $\beta$ es la diferencia en el intercepto $\alpha$ entre el grupo A y el grupo B. Sin variables de control, el valor estimado de $\beta$ sería igual a la diferencia entre las medias de ambos grupos.

Si el modelo incluyera dos sets de variables dummy - por ejemplo, la primera indicaría a los grupos de "tratamiento" y "control", y la segunda, a la instrumentación y otorgamiento de la AUH - el modelo sería:

$$
Y_{i t}=\alpha+\beta D_{i}+\gamma M_{i}+\delta M_{i} * D_{i}+X_{i t} \gamma+\varepsilon_{i t}
$$

La interpretación de los coeficientes estimados sería la siguiente:

$\widehat{\alpha}$ : estimación de la media de los no perceptores de AUH en el grupo de control.

$\widehat{\beta}$ : estimación de la diferencia entre las medias del grupo de tratamiento y control para los no perceptores de AUH (efecto principal de tratamiento). 
Rossignolo: Efecto de la Asignación Universal por Hijo en la reducción de disparidades...

$\widehat{\gamma}$ : estimación de la diferencia entre los perceptores y no perceptores de la $\mathrm{AUH}$ (efecto de la AUH sobre las variables seleccionadas).

$\widehat{\delta}$ : estimación de la diferencia para el efecto de tratamiento y control entre los perceptores y no perceptores de la AUH (efecto interacción).

De acuerdo con esta especificación lineal, puede demostrarse que

$$
\left(\overline{Y_{1}^{T}}-\overline{Y_{0}^{T}}\right)-\left(\overline{Y_{1}^{C}}-\overline{Y_{0}^{C}}\right)=\widehat{\delta} .
$$

Este es el modelo básico de diferencias en diferencias o diferencias dobles que ha sido utilizado por investigadores empíricos en una situación en la que haya existido un cambio en la legislación - $\mathrm{O}$ un suceso- que haya afectado a un grupo pero no al otro, y se asumiera que - en la ausencia de la ley - la diferencia entre ambos grupos se hubiera mantenido estable en el tiempo. En este caso, la variable dummy correspondiente a la vigencia de la ley adoptaría los valores $D_{i}=1$ en el período posterior a la ley y $D_{i}=0$ en caso contrario. Esta variable interactúa con la definición de los grupos de tratamiento y control, considerando a los tratados los perceptores potenciales de la AUH, y al grupo de control a los asalariados formales. Si se asume que el valor esperado de la diferencia de los errores es cero, el modelo de diferencias en diferencias proporciona un estimado consistente del efecto de tratamiento. Card (1990) y Card y Krueger (1994) son los antecedentes más renombrados para este tipo de estudios.

$\mathrm{Si}$, por añadidura - y como es el caso de este artículo-, se procura analizar si la instrumentación de la AUH ha contribuido a reducir las diferencias regionales en un conjunto de indicadores seleccionados, se agrega al modelo estimado una variable dummy adicional $Z_{i}$ que considera las diferentes regiones por pares. La ecuación por estimar sería:

$$
\begin{aligned}
Y_{i t}=\alpha+\beta D_{i}+\gamma M_{i}+\delta M_{i} * D_{i}+ & \theta_{1} Z_{i}+\theta_{2} Z_{i} * D_{i}+\theta_{3} Z_{i} * M_{i} \\
& +\theta_{4} Z_{i} * M_{i} * D_{i}+X_{i t} \gamma+\varepsilon_{i t}
\end{aligned}
$$

El coeficiente de interés ahora es $\theta_{4}$, el coeficiente del término de la triple interacción entre las variables dummy. El estimador por mínimos cuadrados 
ordinarios puede expresarse de la siguiente manera, en donde $A$ y $B$ expresan las diferentes regiones por pares:

$$
\left(\overline{Y_{1, A}^{T}}-\overline{Y_{0, A}^{T}}\right)-\left(\overline{Y_{1, A}^{C}}-\overline{Y_{0 . A}^{C}}\right)-\left(\overline{Y_{1, B}^{T}}-\overline{Y_{0, B}^{T}}\right)=\widehat{\theta}_{4}
$$

En consecuencia, el procedimiento elegido es el siguiente: en primer lugar, se computarán los indicadores de desigualdad para cada una de las regiones antes de la introducción de la Asignación Universal por Hijo. En una segunda instancia, se repetirá el procedimiento para las observaciones muestrales de las encuestas posteriores a la introducción de la AUH, contabilizando a los perceptores efectivos. La aplicación del modelo permitiría establecer si los cambios experimentados en términos de brechas regionales de desigualdad, ingresos, y pobreza resultan significativos a partir de la instrumentación del programa.

En forma consistente con la literatura en la temática, en cada una de las estimaciones se incluyó en forma alternada un conjunto de variables de control tanto a nivel de perceptor de ingresos como al interior del hogar, como la percepción de jubilación o pensión, la condición de actividad económica (ocupado, desocupado o inactivo), la cantidad de adultos (mayores de 65 años de edad) y de menores (edad inferior a 18 años) en el hogar, la proporción de varones y mujeres en el hogar, el máximo nivel educativo alcanzado, y la cantidad de población. Para considerar los ciclos de la economía argentina y sus regiones se incluyó adicionalmente la recaudación real del impuesto provincial sobre los Ingresos Brutos, que es un tributo en cascada que está relacionado con la actividad económica de cada provincia dado que su base imponible se relaciona con las ventas de cada jurisdicción.

\section{Impacto de la AUH en los indicadores seleccionados}

En este apartado se presentan los resultados del análisis para el total país y las regiones. El mismo procurará determinar si, a partir de la instrumentación de la $\mathrm{AUH}$, se han reducido las brechas regionales en relación con diferentes indicadores, entre los cuales se encuentran los niveles de ingreso per cápita regionales, la desigualdad de ingresos personales, y las brechas de pobreza e indigencia. 
Rossignolo: Efecto de la Asignación Universal por Hijo en la reducción de disparidades...

La Tabla 1 muestra algunas estadísticas descriptivas de los grupos pre y post AUH para el total país y las regiones una vez estimado presupuestariamente el impacto de la $\mathrm{AUH}^{5}$. Allí se advierte que, en muchos de los casos, el ingreso per cápita familiar, la desigualdad, la pobreza e indigencia ${ }^{6}$ muestran variaciones significativas en el período que sigue a la instrumentación de la AUH.

Dado que la encuesta define seis regiones, se compara cada una de las regiones por pares con las demás. Así, a partir de las seis regiones quedan establecidos quince emparejamientos para comparar los resultados de las regiones previos y posteriores al establecimiento de la AUH. La región base — es decir aquella con la que las demás regiones se comparan — adoptará el valor cero. Así, por ejemplo, el primer grupo de regiones queda constituido por la comparación entre el GBA y NOA; en este caso la variable dummy regional adopta el valor 1 si es NOA, y cero si es GBA.

Para tal fin, se presentan en lo sucesivo las estimaciones de diferencias en diferencias para las variables analizadas, a saber: desigualdad del ingreso per cápita medida a partir de índice de Gini, el nivel de ingreso per cápita por regiones, y el porcentaje de individuos indigentes y pobres por región. Este análisis se presentará para todos los pares de regiones. Las tablas 2 a 5 presentan los resultados de las regresiones condicionadas. La primera fila muestra el resultado de la variable de interés, las diferencias entre regiones.

5 Es importante notar que, dadas las controversias generadas en Argentina en relación con la medición de la inflación, el INDEC advierte que las series estadísticas publicadas con posterioridad a enero 2007 y hasta diciembre 2015 deben ser consideradas con reservas, excepto las que ya hayan sido revisadas en 2016 y su difusión lo consigne expresamente. Con el fin de calcular en términos reales los datos de ingresos de la EPH, en este artículo se utilizó el deflactor del PIB, que fue revisado y publicado en forma posterior a los microdatos de la EPH.

6 Las estadísticas oficiales sobre los valores de la Canasta Básica Alimentaria (CBA), que determina la Línea de Indigencia utilizada para calcular la proporción de personas indigentes, y la Canasta Básica Total (CBT), empleada para computar la Línea de Pobreza necesaria para contabilizar los individuos pobres, han sido cuestionadas seriamente a partir de diciembre 2007. En este artículo se optó por tomar los valores de la CBA y CBT calculados por la Fundación de Investigaciones Económicas Latinoamericanas. 
En la Tabla 2 se presentan los resultados para el Gini de desigualdad del ingreso per cápita familiar. Tomando como base la región del Gran Buenos Aires, si bien la AUH reduce la desigualdad con relación a las demás regiones, la caída resulta ser significativa en comparación con la región Pampeana. Este panorama se repite en la mayoría de las regiones, pudiendo citarse los casos de la disminución de la brecha de desigualdad entre la región Pampeana con el NOA, NEA, Cuyo y Patagonia. Por ejemplo, la brecha en la desigualdad del ingreso per cápita se reduce en 0,036 entre la región Pampeana y la región del Noreste.

Si se evalúa el ingreso per cápita personal de cada una de las regiones en comparación con las demás, puede verse que la introducción de la AUH, en combinación con el resto de las variables explicativas, reduce la brecha de ingresos, Sin embargo, dicha reducción es, en general, estadísticamente no significativa, a excepción del caso de la brecha del nivel de ingreso per cápita entre la región de la Patagonia y el NEA (Tabla 3).

Este resultado puede explicarse por el hecho de que la $\mathrm{AUH}$, si bien mejora el ingreso per cápita de las familias con ingresos bajos en forma relevante, la proporción que representan los beneficiarios del programa es menor a la de otras fuentes de ingresos, como asalariados formales o perceptores de jubilaciones y pensiones, que representan una proporción más significativa de la población La AUH alcanza a una proporción de la población cuyas variaciones de ingresos no alcanzan a compensar las diferencias en ingresos resultantes del resto de las fuentes, como las mencionadas anteriormente De esta manera, la AUH no tiene un efecto de magnitud suficiente como para reducir las brechas de ingresos entre regiones.

$\mathrm{Al}$ evaluar la variación de la indigencia (pobreza extrema) puede verse que, comparando la región del GBA (Gran Buenos Aires) con el resto de las regiones, se advierte una disminución en las diferencias de la proporción de indigentes antes y después de la AUH entre el GBA y el resto de las regiones, siendo esta diferencia significativa en todos los casos (Tabla 4). 
Rossignolo: Efecto de la Asignación Universal por Hijo en la reducción de disparidades...

Tabla 1. Estadisticas descriptivas de las variables objetivo periodo 2004-2016

\begin{tabular}{|c|c|c|c|c|c|c|c|}
\hline Variables & Gran Buenos Aires & NOA & NEA & Cuyo & Pampeana & Patagónica & Total \\
\hline $\begin{array}{l}\text { Ingreso per cápita fami- } \\
\text { liar de mercado }\end{array}$ & 740,58 & 454,35 & 402,43 & 552,65 & 668,22 & 936,75 & 673,40 \\
\hline $\begin{array}{l}\text { Ingreso per cápita fami- } \\
\text { liar post AUH }\end{array}$ & 884,96 & 606,06 & 541,75 & 685,53 & 837,64 & 1145,6 & 825,53 \\
\hline diferencia & $-144,39$ & $-151,72$ & $-139,33$ & $-132,88$ & $-169,42$ & $-208,84$ & $-152,13$ \\
\hline $\mathrm{t}$ & $-8,76$ & $-11,86$ & $-12,07$ & $-8,74$ & $-9,54$ & $-7,94$ & $-9,49$ \\
\hline p-valor & 0,00 & 0,00 & 0,00 & 0,00 & 0,00 & 0,00 & 0,00 \\
\hline Observaciones & $52 / 44$ & $52 / 44$ & $52 / 44$ & $52 / 44$ & $52 / 44$ & $52 / 44$ & $52 / 44$ \\
\hline $\begin{array}{l}\text { Gini Ingreso per cápita } \\
\text { familiar de mercado }\end{array}$ & 0,49 & 0,48 & 0,48 & 0,45 & 0,46 & 0,45 & 0,49 \\
\hline $\begin{array}{l}\text { Gini Ingreso per cápita } \\
\text { familiar post AUH }\end{array}$ & 0,43 & 0,40 & 0,41 & 0,39 & 0,41 & 0,42 & 0,43 \\
\hline diferencia & 0,06 & 0,08 & 0,07 & 0,06 & 0,05 & 0,04 & 0,06 \\
\hline $\mathrm{T}$ & 14,67 & 15,43 & 15,03 & 18,40 & 13,93 & 12,32 & 16,78 \\
\hline p-valor & 0,00 & 0,00 & 0,00 & 0,00 & 0,00 & 0,00 & 0,00 \\
\hline Observaciones & $52 / 44$ & $52 / 44$ & $52 / 44$ & $52 / 44$ & $52 / 44$ & $52 / 44$ & $52 / 44$ \\
\hline $\begin{array}{l}\text { Indigentes Ingreso per } \\
\text { cápita familiar de mer- } \\
\text { cado }\end{array}$ & $9,5 \%$ & $14,5 \%$ & $17,0 \%$ & $10,8 \%$ & $10,0 \%$ & $8,3 \%$ & $10,5 \%$ \\
\hline $\begin{array}{l}\text { Indigentes Ingreso per } \\
\text { cápita familiar post } \\
\text { AUH }\end{array}$ & $6,1 \%$ & $8,1 \%$ & $10,3 \%$ & $6,6 \%$ & $6,3 \%$ & $4,9 \%$ & $6,6 \%$ \\
\hline diferencia & $3,4 \%$ & $6,3 \%$ & $6,7 \%$ & $4,2 \%$ & $3,7 \%$ & $3,4 \%$ & $4,0 \%$ \\
\hline $\mathrm{t}$ & 6,38 & 5,44 & 4,24 & 6,03 & 6,24 & 6,39 & 6,48 \\
\hline p-valor & 0,00 & 0,00 & 0,00 & 0,00 & 0,00 & 0,00 & 0,00 \\
\hline Observaciones & $52 / 44$ & $52 / 44$ & $52 / 44$ & $52 / 44$ & $52 / 44$ & $52 / 44$ & $52 / 44$ \\
\hline $\begin{array}{l}\text { Pobres Ingreso per cápi- } \\
\text { ta familiar de mercado }\end{array}$ & $28,6 \%$ & $39,5 \%$ & $41,9 \%$ & $32,9 \%$ & $29,3 \%$ & $24,5 \%$ & $30,7 \%$ \\
\hline $\begin{array}{l}\text { Pobres Ingreso per cápi- } \\
\text { ta familiar post AUH }\end{array}$ & $19,9 \%$ & $27,1 \%$ & $30,2 \%$ & $23,0 \%$ & $20,1 \%$ & $16,4 \%$ & $21,3 \%$ \\
\hline diferencia & $8,7 \%$ & $12,5 \%$ & $11,8 \%$ & $9,8 \%$ & $9,2 \%$ & $8,1 \%$ & $9,4 \%$ \\
\hline $\mathrm{t}$ & 7,07 & 5,21 & 4,17 & 6,01 & 6,93 & 5,89 & 7,10 \\
\hline p-valor & 0,00 & 0,00 & 0,00 & 0,00 & 0,00 & 0,00 & 0,00 \\
\hline Observaciones & $52 / 44$ & $52 / 44$ & $52 / 44$ & $52 / 44$ & $52 / 44$ & $52 / 44$ & $52 / 44$ \\
\hline
\end{tabular}

Nota: ajustado por precios regionales al segundo semestre de 2006. Valores constantes al cuarto trimestre de 2009, utilizando el deflactor del PIB.

Fuente: elaboración propia a partir de los microdatos de la EPH-INDEC (s. f.). 


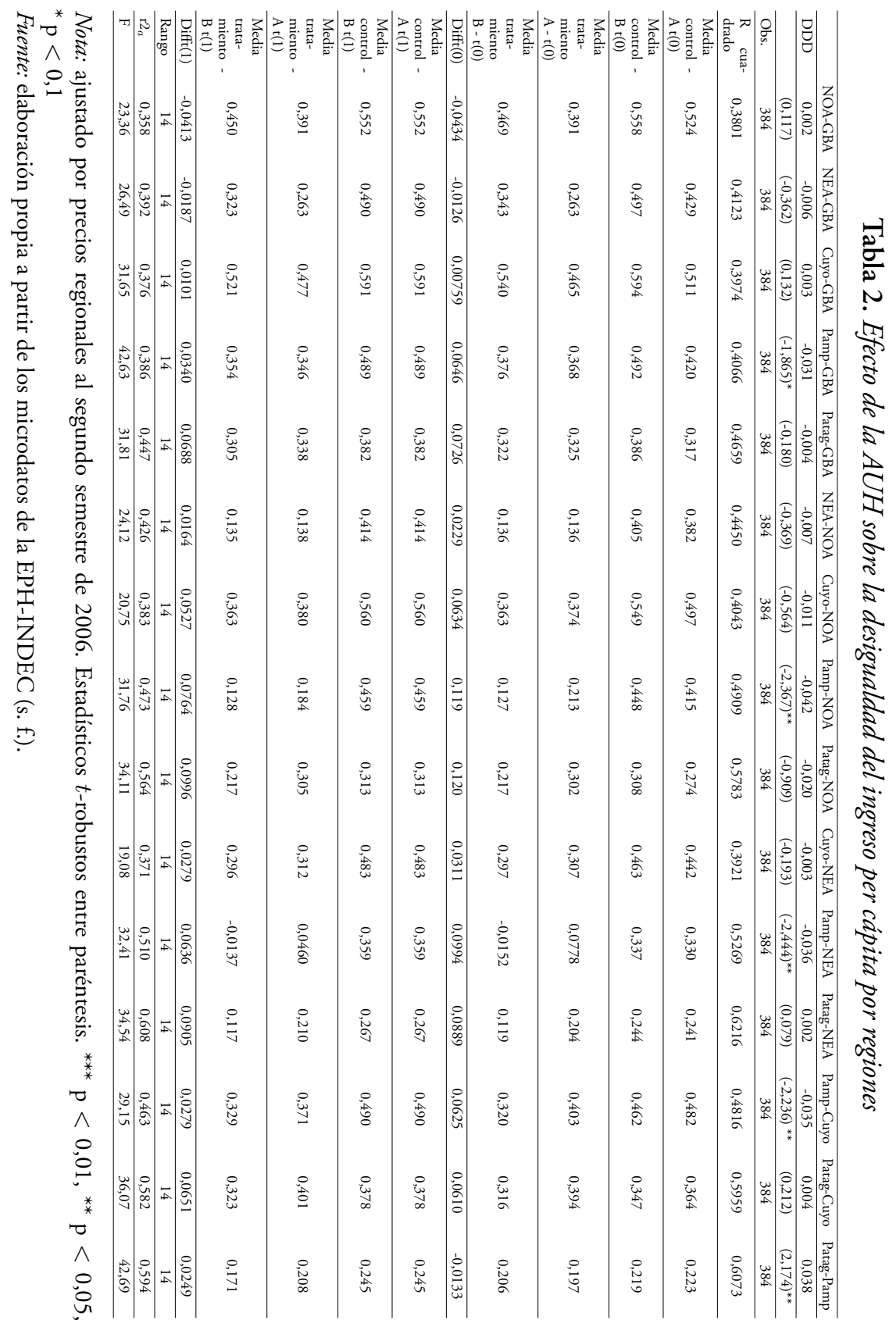


Rossignolo: Efecto de la Asignación Universal por Hijo en la reducción de disparidades...

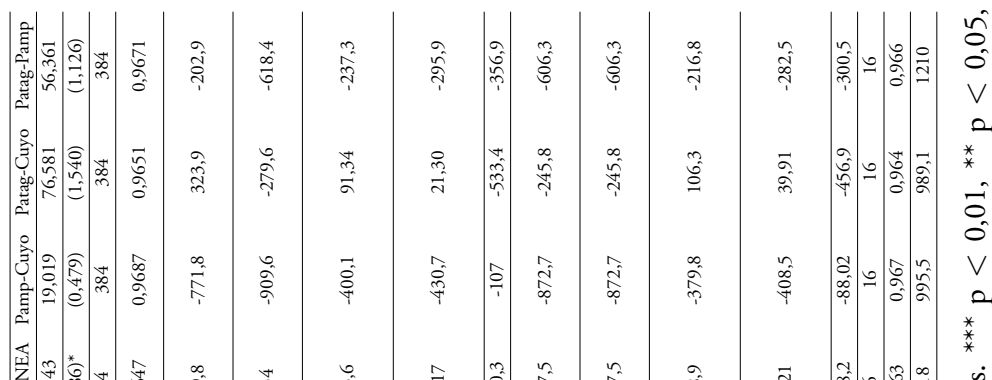

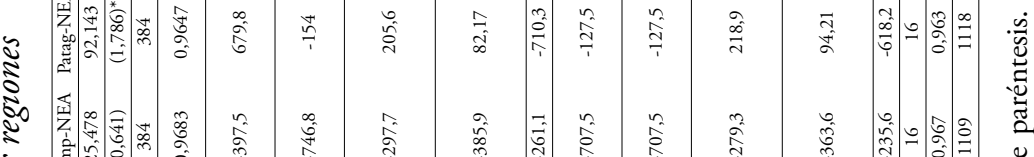

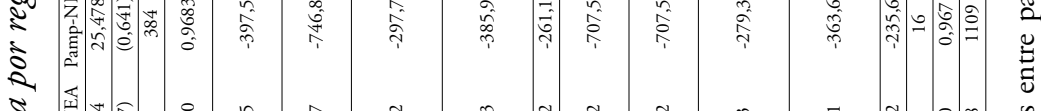

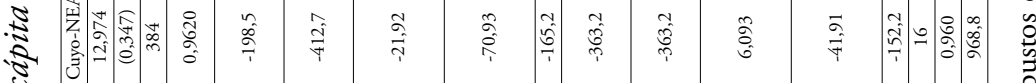

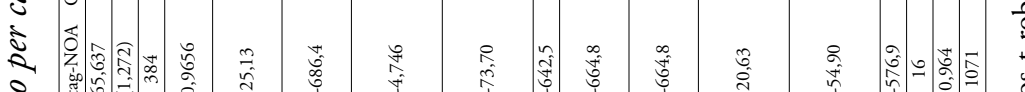

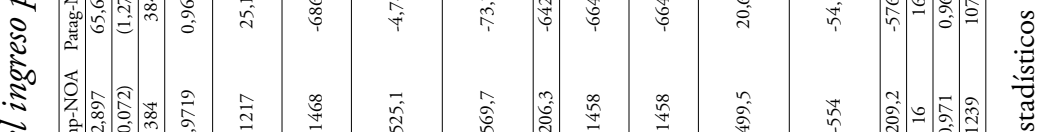

$\stackrel{\dot{4}}{\dot{m}}$

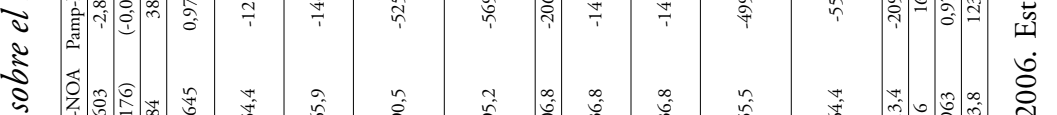

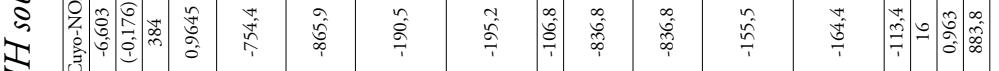

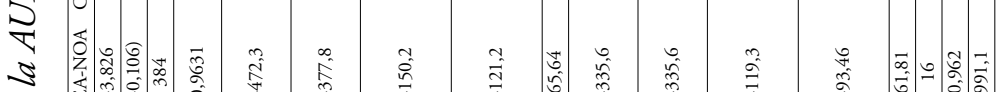

$\frac{1}{2}$

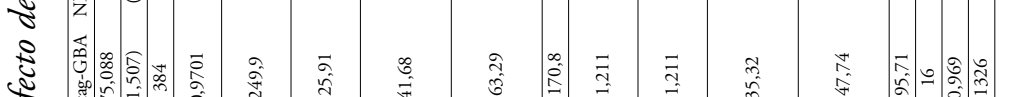

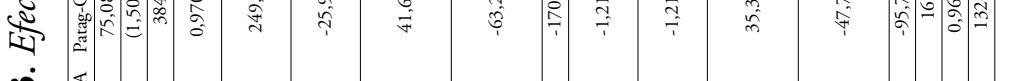

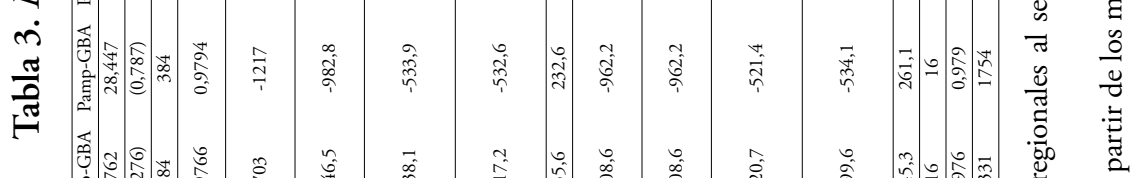

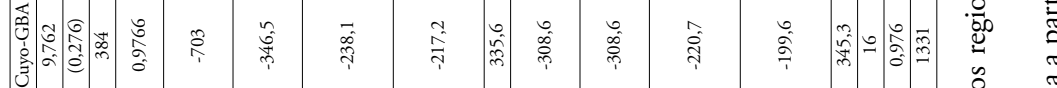

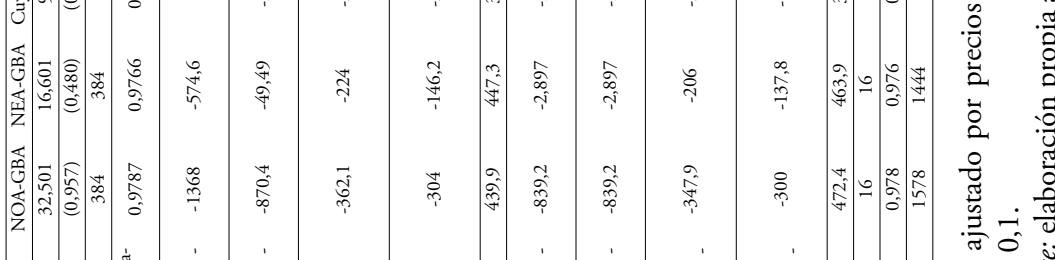

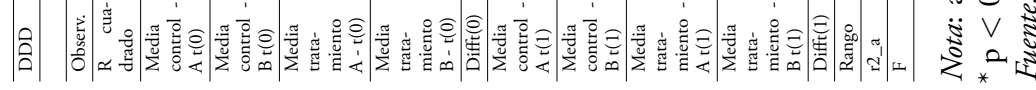




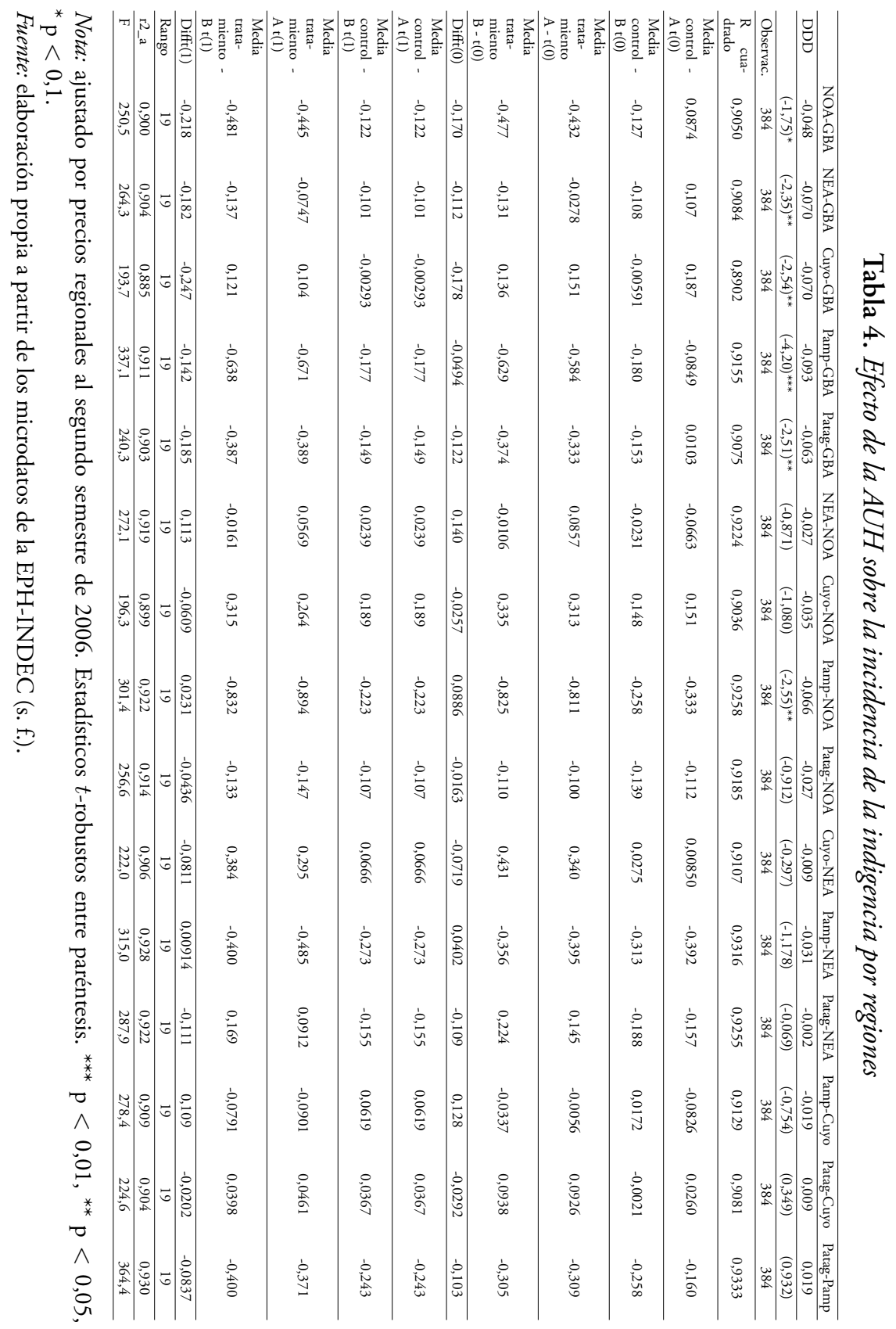


Rossignolo: Efecto de la Asignación Universal por Hijo en la reducción de disparidades...

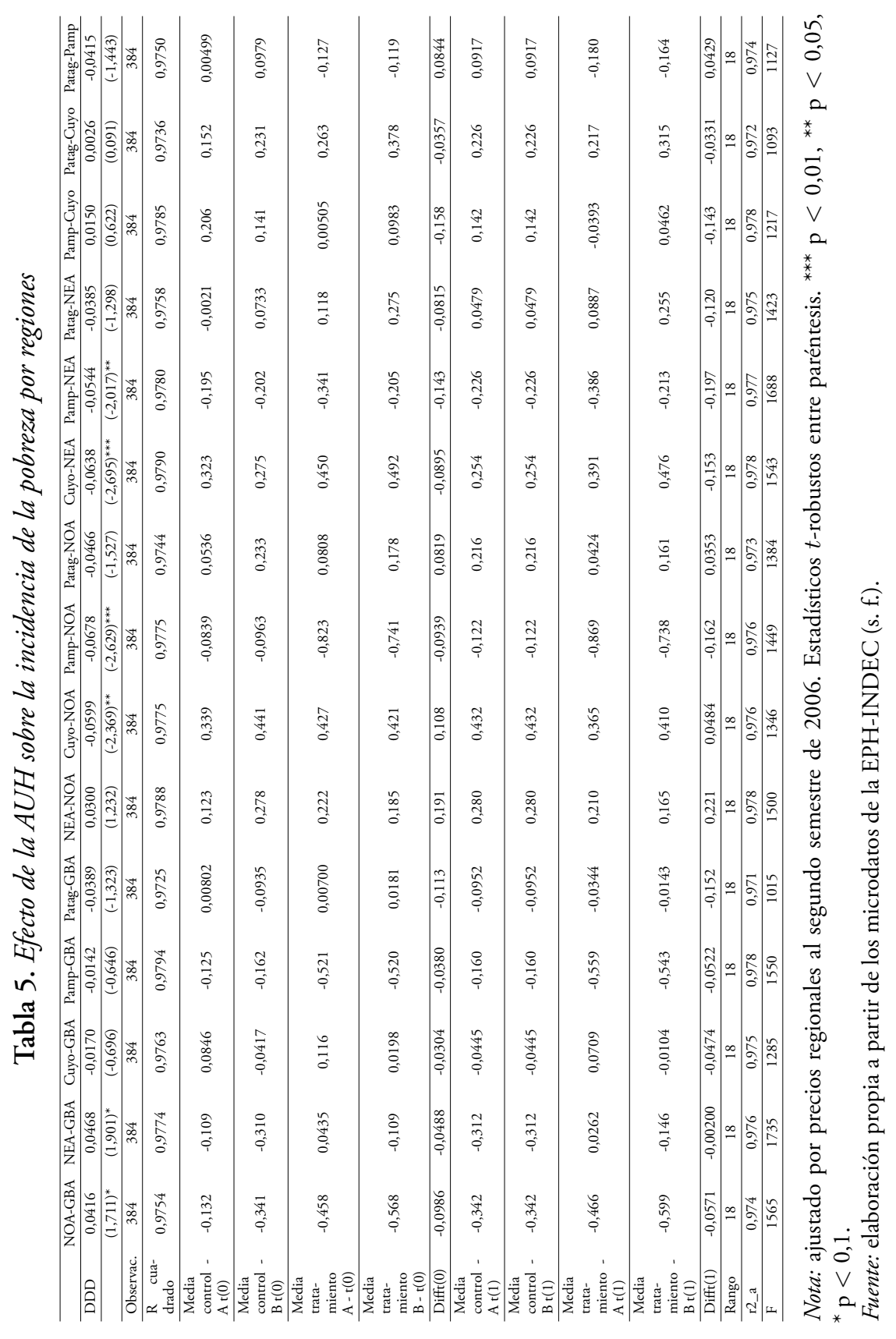


Por ejemplo, la brecha de indigencia entre la región del NEA y del GBA se reduce en un $7 \%$ a partir de la instrumentación de la AUH. La misma conclusión puede obtenerse a partir de analizar la brecha entre la región Pampeana y el GBA, o cuando se comparan las regiones de Cuyo y el NEA con el Gran Buenos Aires.

La significatividad en la reducción de las disparidades no se verifica en el resto de los pares de regiones, a excepción del caso de la disminución en la indigencia para la brecha entre la región Pampeana y el NOA.

Comportamientos similares se advierten al evaluar el impacto de la Asignación Universal por Hijo en la reducción de las brechas de pobreza regionales (Tabla 5). Puede advertirse que, si bien en la mayoría de los casos se advierte una caída en las diferencias entre las proporciones de individuos por debajo de la línea de pobreza por regiones, estas disminuciones no resultan estadísticamente significativas en todos los casos. Las excepciones están constituidas por los casos de las brechas entre la región de Cuyo y la Pampeana con NEA, en donde la brecha se reduce, como en el caso de la brecha entre Cuyoy la Pampeana con la región del NOA.

\section{Conclusiones}

En el año 2009, Argentina aplicó un programa de transferencias de ingresos de carácter nacional denominado Asignación Universal por Hijo destinada a familias con hijos menores de 18 años que se encuentren desocupados o se desempeñen en la economía informal con un ingreso inferior al salario mínimo del sector formal. El programa está basado en un enfoque de protección social dado que equipara las transferencias monetarias percibidas por los trabajadores formales en relación con la cantidad de hijos, de manera que es plausible de permanecer inalterado pese a las potenciales restricciones presupuestarias.

Este programa tiene como objetivo disminuir las desigualdades de ingresos y mitigar el alcance de la indigencia en primer lugar, y de la pobreza en segundo, efectos que han sido estudiados en numerosos trabajos. Adicionalmente, la literatura ha abundado últimamente en el estudio de la fecundidad y oferta de trabajo. 
Rossignolo: Efecto de la Asignación Universal por Hijo en la reducción de disparidades...

Los antecedentes de la literatura han mostrado que la Asignación Universal por Hijo no desincentiva la participación laboral adulta, en general, ni femenina, en particular, como también tiene efectos positivos sobre la escolarización de menores. Además, han documentado el efecto de este programa en la reducción de la pobreza y la desigualdad de ingresos monetarios, sobre todo a nivel nacional.

Sin embargo, la dimensión regional de esta transferencia ha sido evaluada en menor medida. Dado que la AUH consiste en una transferencia nacional cuyos beneficiarios se encuentran en las diferentes regiones geográficas, es interesante establecer si la misma ha resultado de utilidad a los fines de reducir las brechas regionales relacionadas con un conjunto de indicadores regionales agregados. Las transferencias federales focalizadas en los individuos más pobres que conforman en una mayor proporción a las regiones más pobres podrían estar ayudando a reducir las brechas regionales de pobreza y desigualdad en la distribución del ingreso personal y regional.

Para este fin se utilizó como estrategia empírica un modelo de diferencias dobles, a partir de los datos de la EPH. El propósito consistió en analizar si la instrumentación de la $\mathrm{AUH}$ ha servido para reducir las diferencias regionales en niveles de ingreso per cápita, desigualdad, indigencia y pobreza, tomando como momento de instrumentación de la política al período comprendido entre el cuarto trimestre de 2009 y el cuarto trimestre de 2016. Se consideraron como objeto del tratamiento a individuos potencialmente perceptores de esta transferencia y como control a los asalariados formales.

Los resultados que arroja este artículo muestran que, si bien la desigualdad, la pobreza y la indigencia disminuyen con relación al período previo a la instrumentación de la AUH — lo que se verifica en todas las regiones- no puede asegurarse que las brechas regionales de la desigualdad disminuyan en forma estadísticamente significativa en todos los casos. En el caso de las brechas de ingresos, sin embargo, la estimación no arroja resultados estadísticamente significativos sobre la reducción de estas. El efecto de reducción de las brechas regionales se verifica, sin embargo, con mayor significatividad, al analizar los indicadores de pobreza e indigencia. 
Algunas explicaciones a estos resultados pasan, en primer lugar, por la significatividad presupuestaria. Si bien resulta ser un programa de relevancia (en 2016 rondó el 0,6\% del PIB), el mismo representa una magnitud inferior a otros programas, como las jubilaciones y pensiones, que representan una magnitud sustancialmente mayor. En términos fiscales. En segundo lugar, si bien la Asignación Universal por Hijo implicó una sustancial mejora en los indicadores de pobreza, indigencia y desigualdad, según referencian todos los estudios sobre el tema, las diferencias regionales en el número de perceptores potenciales del programa no resultan suficiente para determinar en todos los casos una reducción estadísticamente significativa en las brechas regionales.

\section{Agradecimientos}

Una primera versión de este trabajo fue presentada en las VI Jornadas Iberoamericanas de Financiación Local — financiadas por el Banco Interamericano de Desarrollo y la Comisión Económica para América Latina (CEPAL) - las 52a. Reunión Anual de la Asociación Argentina de Economía Política (2017) en Bariloche, Argentina. Una segunda versión fue presentada en las 52as. Jornadas Internacionales de Finanzas Públicas en Córdoba, Argentina (2019).

\section{Referencias}

Agis, E, Cañete, C., \& Panigo, D. (2013). Empleo, desempleo y politicas de empleo. El impacto de la asignación universal por hijo en Argentina. Centro de Estudios e Investigaciones Laborales (CEIL) y Consejo Nacional de Investigaciones Científicas y Técnicas (CONICET). https://ri.conicet .gov.ar/bitstream/handle/11336/17814/CONICET_Digital_Nro.207 78.pdf? sequence $=1$ \&isAllowed $=y$

Card, D. (1990). The Impact of the Mariel Boatlift on the Miami Labor Market. Industrial and Labor Relations Review, 43(2), 245-257. https: //doi.org/10.1177/001979399004300205

Card, D., \& Krueger, A. (1994). Minimum Wages and Employment: A Case Study of the Fast-Food Industry in New Jersey and Pennsylvania. The 
Rossignolo: Efecto de la Asignación Universal por Hijo en la reducción de disparidades...

American Economic Review, 84(4), 772-793. https://www.jstor.org/st able/2118030

Cont, W., \& Porto, A. (2016). Geografía de la desigualdad en la distribución personal del ingreso y en el impacto distributivo de la politica fiscal. Argentina 1995-2010 Anales de la LI Reunión Anual de la Asociación Argentina de Economía Política. https://aaep.org.ar/anales/works/wor ks2016/cont_porto.pdf

Edo, M., \& Marchionni, M. (2018). Fading out Effect or Long-lasting Nudge? The Impact of a Conditional Cash Transfer Program Beyond Starting the School Year in Argentina [documento de trabajo de CEDLAS No.225]. Universidad Nacional de La Plata. http://sedici .unlp.edu.ar/bitstream/handle/10915/65772/Documento_completo. pdf-PDFA.pdf? sequence $=1$ \&isAllowed $=y$

Edo, M., Marchionni, M., \& Garganta, S. (2015). Conditional Cash Transfer Programs and Enforcement of Compulsory Education Laws. TheCase of Asignación Universal por Hijo in Argentina [documento de trabajo de CEDLAS No. 190]. Universidad Nacional de La Plata. http://sedici.u nlp.edu.ar/bitstream/handle/10915/51621/Documento_completo.pd $\mathrm{f}$ ? sequence $=1$ \&isAllowed $=\mathrm{y}$

Fundación de Investigaciones Económicas Latinoamericanas. (2013). Parte No. 43: Valorización de la Canasta Básica Alimentaria y Canasta Básica Total. http://www.fiel.org/publicaciones/canasta/CAN_BAS_13868 81494189.pdf

Fundación de Investigaciones Económicas Latinoamericanas. (2014a). Parte No. 55: Valorización de la Canasta Básica Alimentaria y Canasta Básica Total. http://www.fiel.org/publicaciones/canasta/CAN_BAS_13892 06969388.pdf

Fundación de Investigaciones Económicas Latinoamericanas. (2014b). Parte No. 64: Valorización de la Canasta Básica Alimentaria y Canasta Básica Total. http://www.fiel.org/publicaciones/canasta/CAN_BAS_14128 83664253.pdf 
Fundación de Investigaciones Económicas Latinoamericanas. (2016). Parte No. 84: Valorización de la Canasta Básica Alimentaria y Canasta Básica Total. http://www.fiel.org/publicaciones/canasta/CAN_BAS_14649 84580715.pdf

Garganta, S., \& Gasparini, L. (2012). El impacto de un programa social sobre la informalidad laboral: el caso de la AUH en Argentina [documento de trabajo de CEDLAS No. 133]. Universidad Nacional de La Plata. http://sedici.unlp.edu.ar/bitstream/handle/10915/18044/Document o_completo__.pdf?sequence $=1$ \&isAllowed $=y$

Garganta, S., Gasparini, L., \& Marchionni, M. (2015). Social Policy and Female Labor Force Participation: the case of AUH in Argentina [conferencia]. Jornadas de la Asociación Argentina de Economía Política (AAEP), Salta.

Garganta, S., Gasparini, L., Marchionni, M., \& Tappatá, M. (2017). The Effect of Cash Transfers on Fertility: Evidence for Argentina. Population Research and Policy Review, 36 (1), 1-24. http://sedici.unlp.edu.ar/bitst ream/handle/10915/104754/Documento_completo.pdf?sequence=1

Higgins, S, \& Lustig, N. (2017). Allocating Taxes and Transfers and Constructing Income Concepts. En N. Lustig (ed.), Commitment to Equity Handbook. A Guide to Estimating the Impact of Fiscal Policy on Inequality and Poverty. Brookings Institution and CEQ Institute.

Higgins, S., \& Pereira, C. (2013). The Effects of Brazil's High Taxation and Social Spendingon The Distribution of Household Income [CEQ Working Paper No. 7]. Tulane University. https://commitmentoequity.org/publ ications-ceqworkingpapers/

Instituto Nacional de Estadística y Censos (INDEC). (s. f.). Encuesta Permante de Hogares (EPH). Consultado el 30 de enero de 2021. https: //www.indec.gob.ar/indec/web/Institucional-Indec-BasesDeDatos

Lustig, N., \& Pessino, C. (2013). Social Spending and Income Redistribution in Argentina in the 2000s: The Rising Role of Noncontributory Pensions 
[CEQ Institute Working Paper No. 5]. Tulane University. https://co mmitmentoequity.org/publications-argentina/

Maurizio, R. (2009). Políticas de transferencias monetarias en Argentina: Evaluación de su impacto sobre la pobreza y la desigualdad, y evaluación de sus costos. En S. N. Keifman (comp.), Reflexiones y propuestas para mejorar la distribución del ingreso en Argentina (pp. 161-266). Oficina de la Organización Internacional del Trabajo en Buenos Aires.

Maurizio, R., \& Vázquez, G. (2014). Argentina: efectos del programa Asignación Universal por Hijo en el comportamiento laboral de los adultos. Revista de la CEPAL, 113, 121-144. https://repositorio.ce pal.org/bitstream/handle/11362/36962/RVE113MaurizioVázquez.p df? sequence $=1$

Roca, E. (2011). Asignación Universal por Hijo (AUH): extensión de las asignaciones familiares. Revista Debate Público. Reflexión de Trabajo Social, 1(1). 29-43. http://trabajosocial.sociales.uba.ar/wp-content /uploads/sites/13/2016/03/6_roca.pdf

Rossignolo, D. (2016). Taxes, Expenditures, Poverty, and Income Distribution in Argentina [CEQ Institute Working Paper No. 45]. Tulane University. https://commitmentoequity.org/publications_files/Argentina/CEQ _WP45_TAXES_EXPENDITURES_POVERTY_AND_INCOM E_DISTRIBUTION_IN_ARGENTINA.pdf

Scott, J., De la Rosa, E., \& Aranda, R. (2017). Inequality and Fiscal Redistribution in Mexico 1992-2015 [WIDER Working Paper 194/2017]. United Nations University. https://www.wider.unu.edu/publication/inequali ty-and-fiscal-redistribution-mexico 